\title{
Epidemiologe erhält Staatsmedaille
}

rof. Dr. Dr. Heinz-Erich Wichmann, Direktor des Instituts für Epidemiologie am GSF-Forschungszentrum für Umwelt und Gesundheit und Professor am Lehrstuhl für Epidemiologie an der Ludwig-Maximilians-Universität, München, wurde die Staatsmedaille für Umwelt und Gesundheit des Freistaates Bayern verliehen. Mit dieser Auszeichnung würdigt der bayerische Staat Personen, die sich besondere Verdienste um den Natur- und Umweltschutz, um Gesundheit bzw. um den Schutz der Verbraucher erworben haben.

Der bayerische Staatsminister für Umwelt, Gesundheit und Verbraucherschutz, Dr. Werner Schnappauf, hob in seiner Laudatio hervor, dass Wichmann einer der international renommiertesten deutschen Epidemiologen sei. Während seiner 30-jährigen Forschungslaufbahn habe er zahlreiche Studien im Bereich der Umweltepidemiologie durchgeführt, deren Schwerpunkt auf den Auswirkungen von Luftschadstoffen auf die Ge- sundheit lagen. Diese dienten als Grundlage der Gesetzgebung. Er habe darüber hinaus große bevölkerungsbezogene Kohortenstudien konzipiert, die Aufschlüsse über aktuelle Gesundheitsfragen lieferten, und er hat als einer der ersten in Deutschland den Einfluss der genetischen Prädisposition als krankheitsfördernden Faktor im Rahmen von GenUmwelt-Interaktionen untersucht. $g s f$

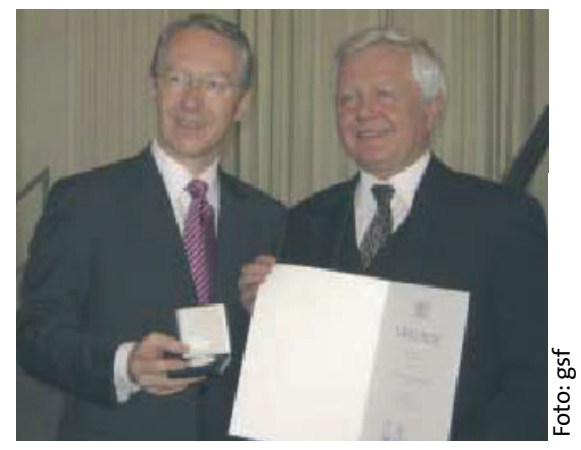

Dr. Werner Schnappauf (links) überreichte am 28. Oktober 2005 die Staatsmedaille an Prof. Dr. Dr. Wichmann.
Jubiläumsfortbildung im ADIZ

Am 24. Juli 1906 erblickte das Wort „Allergie" das Licht der Welt - in gedruckter Form in der Münchner

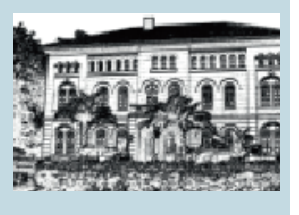
Medizinischen Wochenschrift. Vater des Begriffs war der Wiener Pädiater Clemens von Pirquet. Im Jahr 2006 wird aber nicht nur das Wort Allergie 100 Jahre alt, auch das Allergie-Dokumentations- und Informationszentrum (ADIZ) in Bad Lippspringe feiert sein 15-jähriges Bestehen. Aus Anlass dieses Doppeljubiläums veranstaltet das ADIZ am 26. April 2006 die zertifizierte Fortbildungsveranstaltung „100 Jahre Allergie - 15 Jahre ADIZ“.

Thema sind Allergien im Spiegel der Zeit. Die Veranstaltung steht unter der Schirmherrschaft der Deutschen Gesellschaft für Allergologie und klinische Immunologie.

\section{Susanne Böning}

ADIZ im Prinzenpalais,

Arminiuspark 11, 33175 Bad Lippspringe

Tel.: (o 52 52) 954502

Fax: (o 52 52) 954501

E-Mail: adizf@aol.com 